University of Nebraska - Lincoln

DigitalCommons@University of Nebraska - Lincoln

\title{
Empirical Studies of a Prediction Model for Regression Test Selection
}

\author{
Mary Jean Harrold \\ Georgia Institute of Technology \\ David Rosenblum \\ University of California, Irvine \\ Gregg Rothermel \\ University of Nebraska-Lincoln, gerother@ncsu.edu \\ Elaine Weyuker \\ AT\&T Labs
}

Follow this and additional works at: https://digitalcommons.unl.edu/csearticles

Part of the Computer Sciences Commons

Harrold, Mary Jean; Rosenblum, David; Rothermel, Gregg; and Weyuker, Elaine, "Empirical Studies of a Prediction Model for Regression Test Selection" (2001). CSE Journal Articles. 10.

https://digitalcommons.unl.edu/csearticles/10

This Article is brought to you for free and open access by the Computer Science and Engineering, Department of at DigitalCommons@University of Nebraska - Lincoln. It has been accepted for inclusion in CSE Journal Articles by an authorized administrator of DigitalCommons@University of Nebraska - Lincoln. 


\title{
Empirical Studies of a Prediction Model for Regression Test Selection
}

\author{
Mary Jean Harrold, Member, IEEE Computer Society, David Rosenblum, Senior Member, IEEE, \\ Gregg Rothermel, Member, IEEE Computer Society, and Elaine Weyuker, Senior Member, IEEE
}

\begin{abstract}
Regression testing is an important activity that can account for a large proportion of the cost of software maintenance. One approach to reducing the cost of regression testing is to employ a selective regression testing technique that 1) chooses a subset of a test suite that was used to test the software before the modifications, then 2) uses this subset to test the modified software. Selective regression testing techniques reduce the cost of regression testing if the cost of selecting the subset from the test suite together with the cost of running the selected subset of test cases is less than the cost of rerunning the entire test suite. Rosenblum and Weyuker recently proposed coverage-based predictors for use in predicting the effectiveness of regression test selection strategies. Using the regression testing cost model of Leung and White, Rosenblum and Weyuker demonstrated the applicability of these predictors by performing a case study involving 31 versions of the KornShell. To further investigate the applicability of the Rosenblum-Weyuker (RW) predictor, additional empirical studies have been performed. The RW predictor was applied to a number of subjects, using two different selective regression testing tools, DejaVu and TestTube. These studies support two conclusions. First, they show that there is some variability in the success with which the predictors work and second, they suggest that these results can be improved by incorporating information about the distribution of modifications. It is shown how the RW prediction model can be improved to provide such an accounting.
\end{abstract}

Index Terms-Software maintenance, regression testing, selective retest, regression test selection.

\section{INTRODUCTION}

$\mathrm{R}_{\mathrm{a}}$ egression testing is an important activity that can ccount for a large proportion of the cost of software maintenance [5], [17]. Regression testing is performed on modified software to provide confidence that the software behaves correctly and that modifications have not adversely impacted the software's quality. One approach to reducing the cost of regression testing is to employ a selective regression testing technique. A selective regression testing technique chooses a subset of a test suite that was used to test the software before modifications were made, and then uses this subset to test the modified software. ${ }^{1}$ Selective regression testing techniques reduce the cost of regression testing if the cost of selecting the subset from the test suite

1. A variety of selective regression testing techniques have been proposed (e.g., [1], [3], [4], [6], [7], [8], [9], [10], [12], [13], [15], [16], [18], [21], [26], [27], [28], [29], [30]). For an overview and analytical comparison of these techniques, see [25]

- M.J. Harrold is with the College of Computing, Georgia Institute of Technology, 801 Atlantic Dr., Atlanta, GA 30332-0280. E-mail: harrold@cc.gatech.edu.

- D. Rosenblum is with the Department of Information and Computer Science Institute for Software Research, University of California, Irvine, ICS2 209, Irvine, CA 92697-3425. E-mail: dsr@ics.uci.edu.

- E. Weyuker is with ATET Labs-Research, Room E237, 180 Park Ave., Florham Park, NJ 07932. E-mail: weyuker@research.att.com.

- G. Rothermel is with the Department of Computer Science, Oregon State University, Dearborn Hall 307-A, Corvallis, OR 97331.

E-mail: grother@cs.orst.edu.

Manuscript received 23 Feb. 1998; revised 19 Aug. 1999; accepted 15 Sept. 1999.

Recommended for acceptance by M. Young.

For information on obtaining reprints of this article, please send e-mail to: tse@computer.org, and reference IEEECS Log Number 106365. together with the cost of running the selected subset of test cases is less than the cost of rerunning the entire test suite.

Empirical results obtained by Rothermel and Harrold on the effectiveness of their selective regression testing algorithms, implemented as a tool called DejaVu, suggest that test selection can sometimes be effective in reducing the cost of regression testing by reducing the number of test cases that need to be rerun [24], [26]. However, these studies also show that there are situations in which their algorithm is not cost-effective. Furthermore, other studies performed independently by Rosenblum and Weyuker with a different selective regression testing algorithm, implemented as a tool called Test Tube [8], also show that such methods are not always cost-effective [23]. When selective regression testing is not cost-effective, the resources spent performing the test case selection are wasted. Thus, Rosenblum and Weyuker argue in [23] that it would be desirable to have a predictor that is inexpensive to apply but could indicate whether or not using a selective regression testing method is likely to be worthwhile.

With this motivation, Rosenblum and Weyuker [23] propose coverage-based predictors for use in predicting the cost-effectiveness of selective regression testing strategies. Their predictors use the average percentage of test cases that execute covered entities-such as statements, branches, or functions-to predict the number of test cases that will be selected when a change is made to those entities. One of these predictors is used to predict whether a safe selective regression testing strategy (one that selects all test cases that cover affected entities) will be cost-effective. Using the regression testing cost model of Leung and White [19], Rosenblum and Weyuker demonstrate the usefulness of this 
predictor by describing the results of a case study they performed involving 31 versions of the KornShell [23]. In that study, the predictor reported that, on average, it was expected that 87.3 percent of the test cases would be selected. Using the TestTube approach, 88.1 percent were actually selected on average over the 31 versions. Since the difference between these values is very small, the predictor was clearly extremely accurate in this case. The authors explain, however, that because of the way their selective regression testing model employs averages, the accuracy of their predictor might vary significantly in practice from version to version. This is particularly an issue if there is a wide variation in the distribution of changes among entities [23]. However, because their predictor is intended to be used for predicting the long-term behavior of a method over multiple versions, they argue that the use of averages is acceptable.

To further investigate the applicability of the RosenblumWeyuker (RW) predictor for safe selective regression testing strategies, we present in this paper the results of additional studies. We applied the RW predictor to subjects developed by researchers at Siemens Corporate Research for use in studies to compare the effectiveness of certain software testing strategies [14]. For the current paper, we used both DejaVu and TestTube to perform selective regression testing. In the following sections, we discuss the results of our studies.

\section{Background: The Rosenblum-Weyuker PRedictor}

Rosenblum and Weyuker presented a formal model of regression testing to support the definition and computation of predictors of cost-effectiveness [23]. Their model builds on work by Leung and White on modeling the cost of employing a selective regression testing method [19]. In both models, the total cost of regression testing incorporates two factors: the cost of executing test cases and the cost of performing analyses to support test selection. A number of simplifying assumptions are made in the representation of the cost in these models:

1. The costs are constant on a per-test-case basis.

2. The costs represent a composite of the various costs that are actually incurred; for example, the cost associated with an individual test case is a composite that includes the costs of executing the test case, storing execution data, and validating the results.

3. The cost of the analyses needed to select test cases from the test suite has a completely negative impact on cost-effectiveness, in the sense that analysis activities drain resources that could otherwise be used to support the execution of additional test cases.

4. Cost-effectiveness is an inherent attribute of test selection over the complete maintenance life-cycle, rather than an attribute of individual versions.

As in Rosenblum and Weyuker's model [23], we let $P$ denote the system under test and let $T$ denote the regression test suite for $P$, with $|T|$ denoting the number of individual test cases in $T$. Let $M$ be the selective regression testing method used to choose a subset of $T$ for testing a modified version of $P$ and let $E$ be the set of entities of the system under test that are considered by $M$. It is assumed that $T$ and $E$ are nonempty and that every syntactic element of $P$ belongs to at least one entity in $E$.

The Rosenblum-Weyuker (RW) model defined covers $_{M}(t, e)$ as the coverage relation induced by method $M$ for $P$ and defined over $T \times E$, with $\operatorname{covers}_{M}(t, e)$ true if and only if the execution of $P$ on test case $t$ causes entity $e$ to be exercised at least once. Rosenblum and Weyuker specify meanings for "exercised" for several kinds of entities of $P$. For example, if $e$ is a function or module of $P, e$ is exercised whenever it is invoked; if $e$ is a simple statement, statement condition, definition-use association, or other kind of execution subpath of $P, e$ is exercised whenever it is executed.

Letting $E^{\mathcal{C}}$ denote the set of covered entities, the RW model defined $E^{\mathcal{C}}$ as follows:

$$
E^{\mathcal{C}}=\left\{e \in E \mid \exists t \in T\left(\operatorname{covers}_{M}(t, e)\right)\right\},
$$

with $\left|E^{\mathcal{C}}\right|$ denoting the number of covered entities. Furthermore, $\operatorname{cover}_{M}(t, e)$ can be represented by a 0-1 matrix $C$, whose rows represent elements of $T$ and whose columns represent elements of $E$. Then, element $C_{i, j}$ of $C$ is defined to be:

$$
C_{i, j}= \begin{cases}1 & \text { if } \operatorname{covers}_{M}(i, j) \\ 0 & \text { otherwise }\end{cases}
$$

Finally, $C C$ was the cumulative coverage achieved by $T$ (i.e., the total number of ones in the 0-1 matrix):

$$
C C=\sum_{i=1}^{|T|} \sum_{j=1}^{|E|} C_{i, j} .
$$

As a first step in computing a predictor for safe strategies when a single entity had been changed, Rosenblum and Weyuker considered the expected number of test cases that would have to be rerun. Calling this average $N_{M}$, they defined:

$$
N_{M}=\frac{C C}{|E|} .
$$

Rosenblum and Weyuker emphasized that this predictor was only intended to be used when the selective regression testing strategy's goal was to rerun all affected test cases.

A slightly refined variant of $N_{M}$ was defined using $E^{\mathcal{C}}$ rather than $E$ as the universe of entities.

$$
N_{M}^{\mathcal{C}}=\frac{C C}{\left|E^{\mathcal{C}}\right|} .
$$

Then, the fraction of the test suite that must be rerun was denoted $\pi_{M}$, the predictor for $\left|T_{M}\right| /|T|$ :

$$
\begin{aligned}
\pi_{M} & =\frac{N_{M}^{\mathcal{C}}}{|T|} \\
& =\frac{C C}{\left|E^{\mathcal{C}}\right||T|} .
\end{aligned}
$$

Rosenblum and Weyuker discussed results of a case study in which test selection and prediction results were compared for 31 versions of the KornShell using 
the TestTube selective regression testing method. As mentioned above, in this study, the test selection technique chose an average of 88.1 percent of the test cases in the test suite over the 31 versions, while the predicted value was 87.3 percent. They concluded that, because the difference between these values was very small, their results indicated the usefulness of their predictor as a way of predicting cost-effectiveness.

\section{Two New Empirical Studies of the Rosenblum-Weyuker Predictor}

The results of the Rosenblum-Weyuker case study were encouraging for two reasons:

1. The difference between the predicted and actual values was insignificant.

2. Because a large proportion of the test set would have to be rerun for regression testing, and it could be quite expensive to perform the analysis necessary to determine which test cases did not need to be rerun; it would often be cost-effective to use the predictor to discover this and then simply rerun the entire test suite rather than selecting a subset of the test suite.

Nevertheless, this study involved a single selective regression testing method applied to a single subject program, albeit a large and widely-used one for which there were a substantial number of actual production versions. In order to obtain a broader picture of the usefulness of the RW predictor, we conducted additional studies with other subject software and other selective regression testing methods. In particular, we performed two new studies with two methods, DejaVu and TestTube, applied to a suite of subject programs that have been used in other studies in the testing literature.

\subsection{Statement of Hypothesis}

The hypothesis we tested in our new studies is the hypothesis of the Rosenblum-Weyuker study:

Hypothesis. Given a system under test $P$, a regression test suite $T$ for $P$, and a selective regression testing method $M$, it is possible to use information about the coverage relation cover $_{M}$ induced by $M$ over $T$ and the entities of $P$ to predict whether or not $M$ will be cost-effective for regression testing future versions of $P$.

The previous and current studies test this hypothesis under the following assumptions:

1. The prediction is based on a cost metric that is appropriate for $P$ and $T$. Certain simplifying assumptions are made about costs, as described in Section 2.

2. The prediction is performed using data from a single version of $P$ to predict cost-effectiveness for all future versions of $P$.

3. "Cost-effective" means that the cumulative cost over all future versions of $P$ of applying $M$ and executing the test cases in $T$ selected by $M$ is less than the cumulative cost over all future versions of $P$ of running all test cases in $T$ (the so-called retest-all method).

\subsection{Subject Programs}

As subjects for our new studies, we used seven $C$ programs that had been previously used in a study by researchers at Siemens Corporate Research [14]. Because the researchers at Siemens sought to study the fault-detecting effectiveness of different coverage criteria, they created faulty modified versions of the seven base programs by manually seeding the programs with faults, usually by modifying a single line of code in the base version, and never modifying more than five lines of code. Their goal was to introduce faults that were as realistic as possible, based on their experience. Ten people performed the fault seeding, working "mostly without knowledge of each other's work" [14, p. 196].

For each base program, Hutchins et al. created a large test pool containing possible test cases for the program. To populate these test pools, they first created an initial set of black-box test cases "according to good testing practices, based on the tester's understanding of the program's functionality and knowledge of special values and boundary points that are easily observable in the code" [14, p. 194], using the category partition method and the Siemens Test Specification Language tool [2], [20]. They then augmented this set with manually-created white-box test cases to ensure that each executable statement, edge, and definition-use pair in the base program or its control flow graph was exercised by at least 30 test cases. To obtain meaningful results with the seeded versions of the programs, the researchers retained only faults that were "neither too easy nor too hard to detect" [14, p. 196], which they defined as being detectable by at least three and at most 350 test cases in the test pool associated with each program.

Table 1 presents information about these subjects. For each program, the table lists its name, the number of lines of code in the program, the number of functions in the program, the number of modified (i.e., fault seeded) versions of the program, the size of the test pool, the average number of test cases in each of the 1,000 coveragebased test suites we generated for our studies, and a brief description of the program's function. We describe the generation of the 1,000 coverage-based test suites in greater detail below.

\subsection{Design of the New Studies}

In both studies, our analysis was based on measurements of the following variables:

Independent Variable. For each subject program $P$, test suite $T$ for $P$, and selective regression testing method $M$, the independent variable is the relation $\operatorname{cover}_{M}(t, e)$ defined in Section 2.

Dependent Variables. For each subject program $P$, test suite $T$ for $P$, and selective regression testing method $M$, there are two dependent variables: 1) the cost of applying $M$ to $P$ and $T$ and 2 ) the cost of executing $P$ on the test cases selected by $M$ from $T$.

We then used the model described in Section 2 plus our measurements of the dependent variables to perform analyses of cost-effectiveness. Each study involved different kinds of analysis, as described in the following: 
TABLE 1

Summary of Subject Programs

\begin{tabular}{|c|c|c|c|c|c|c|}
\hline Program & $\begin{array}{c}\text { Lines of } \\
\text { Code }\end{array}$ & $\begin{array}{c}\text { Number of } \\
\text { Functions }\end{array}$ & $\begin{array}{c}\text { Number of } \\
\text { Modified Versions }\end{array}$ & $\begin{array}{c}\text { Test Pool } \\
\text { Size }\end{array}$ & $\begin{array}{c}\text { Average Test } \\
\text { Suite Size }\end{array}$ & Description \\
\hline tcas & 138 & 9 & 41 & 1608 & 6 & altitude separation \\
schedule2 & 297 & 16 & 10 & 2710 & 8 & priority scheduler \\
schedule1 & 299 & 18 & 9 & 2680 & 8 & priority scheduler \\
totinfo & 346 & 7 & 23 & 1054 & 7 & information measure \\
printtokens2 & 483 & 19 & 10 & 4115 & 12 & lexical analyzer \\
printtokens1 & 402 & 20 & 7 & 4130 & 16 & lexical analyzer \\
replace & 516 & 21 & 32 & 5542 & 19 & pattern replacement \\
\hline
\end{tabular}

For both studies, we used the Siemens test pools from which we selected smaller test suites. In particular, we randomly generated 1,000 branch-coverage-based test suites for each base program from its associated test pool. ${ }^{2}$ To create each test suite $T_{i}, 1 \leq i \leq 1,000$, for program $P$, we applied the following algorithm:

1. Initialize $T_{i}$ to $\phi$.

2. While uncovered coverable branches remain,
a. randomly select an element $t$ from $T$ using a uniform distribution,
b. execute $P$ with $t$, recording coverage,
c. add $t$ to $T_{i}$ if it covered branches in $P$ not previously covered by the other test cases in $T_{i}$,

3. If $T_{i}$ differs from all other previously generated test suites, keep $T_{i}$, and increment $i$; otherwise, generate a new $T_{i}$.

Step 3 of the procedure ensures that there are no duplicate test suites for a program, although two different test suites may have some test cases in common.

For both of our studies, we computed the cost measures (dependent variables) for both DejaVu and TestTube, and we compared this information to test selection predictions computed using the RW predictor. To gather this information, we considered each base program $P$ with each modified version $P_{i}$ and each test suite $T_{j}$. For each $P$ and each $T_{j}$, we computed the following:

$\pi_{\mathrm{DejaVu}_{j}}$ : The percentage of test cases of $T_{j}$ that the

RW predictor predicts will be selected by DejaVu when an arbitrary change is made to $P$.

$\pi_{\text {TestTube }}$ : The percentage of test cases of $T_{j}$ that the RW predictor predicts will be selected by TestTube when an arbitrary change is made to $P$.

$S_{\mathrm{DejaVu}_{i, j}}$ : The percentage of test cases of $T_{j}$ actually selected by DejaVu for the changes made to create $P_{i}$ from $P$.

$S_{\text {TestTube }_{i, j}}$ : The percentage of test cases of $T_{j}$ actually selected by TestTube for the changes made to create $P_{i}$ from $P$.

Finally, we used these values to evaluate the accuracy of the RW predictor, as described in detail in the following sections.

2. Because our studies focused on the cost-effectiveness of selective regression testing methods rather than the fault-detecting effectiveness of coverage criteria, the realism of the modifications made to the Siemens programs is not a significant issue. What is more important is that they were made independently by people not involved in our study, thereby reducing the potential for bias in our results.

\subsubsection{Study 1}

The goal of our first study was to determine the accuracy, on average, of the RW predictor for the subject programs, modified versions, and test suites for each of the selective regression testing approaches we considered. Therefore, we used the regression test selection information described above to compute the average percentages of test cases selected by DejaVu and TestTube over all versions $P_{i}$ of $P$. For each $P$ and each $T_{j}$, we computed the following:

$$
\begin{aligned}
\bar{S}_{\text {DejaVu }_{j}} & =\frac{\sum_{i=1}^{\mid \text {versions of } P \mid} S_{\mathrm{DejaVu}_{i, j}}}{\mid \text { versions of } P \mid}, \\
\bar{S}_{\text {TestTube }_{j}} & =\frac{\sum_{i=1}^{\mid \text {versions of } P \mid} S_{\text {TestTube }_{i, j}}}{\mid \text { versions of } P \mid} .
\end{aligned}
$$

The first step in our investigation was to see how much the percentage of test cases actually selected by each of the methods differed from the predicted percentage of test cases. For this analysis, we needed the following two additional pieces of data, which we computed for each $P$ and each $T_{j}$ :

$$
\begin{gathered}
\bar{D}_{\text {DejaVu }_{j}}=\pi_{\text {DejaVu }_{j}}-\bar{S}_{\text {DejaVu }_{j}}, \\
\bar{D}_{\text {TestTube }_{j}}=\pi_{\text {TestTube }_{j}}-\bar{S}_{\text {TestTube }_{j}} .
\end{gathered}
$$

$\bar{D}_{\mathrm{DejaVu}_{j}}$ and $\bar{D}_{\text {TestTube }_{j}}$ represent the deviations of the percentages of the test cases predicted by the RW predictor for $T_{j}$ from the average of the actual percentages of test cases selected by the respective method for all versions $P_{i}$ of $P$. Because it is possible for $\bar{D}_{\mathrm{DejaVu}_{j}}$ and $\bar{D}_{\text {TestTube }_{j}}$ to lie anywhere in the range of $[-100,100]$, we wanted to determine the ranges into which the values for $\bar{D}_{\mathrm{DejaVu}_{j}}$ and $\bar{D}_{\text {TestTube }_{j}}$ actually fell. Thus, we rounded the values of $\bar{D}_{\mathrm{DejaVu}_{j}}$ and $\bar{D}_{\text {TestTube }_{j}}$ to the nearest integer $I$ and computed, for each $I$ (such that $-100 \leq I \leq 100$ ), the percentage of the rounded $\bar{D}$ values with value $I$. For each $P$, using each of its $\bar{D}_{\mathrm{DejaVu}_{j}}$ values, the result was a set $\bar{H}_{\text {DejaVu }}$ :

$$
\begin{gathered}
\bar{H}_{\text {DejaVu }}=\{(r, p r d) \mid r \text { is the range value, }-100 \leq r \leq 100, \\
\text { prd is the percentage of rounded } \left.\bar{D}_{\text {DejaVu }_{j}} \text { values at } r\right\} .
\end{gathered}
$$


Similarly, for each $P$, using each of its $\bar{D}_{\text {TestTube }_{j}}$ values, the result was a set $\bar{H}_{\text {TestTube: }}$

$$
\begin{gathered}
\bar{H}_{\text {TestTube }}=\{(r, p r d) \mid r \text { is the range value, }-100 \leq r \leq 100, \\
\text { prd is the percentage of rounded } \bar{D}_{\text {TestTube } \left._{j} \text { values at } r\right\} .}
\end{gathered}
$$

These sets essentially form a histogram of the deviation values. In the ideal case of perfect prediction, all deviations would be zero and, therefore, each graph would consist of the single point $(0,100$ percent).

\subsubsection{Study 2}

In Study 1, we treated the RW predictor as a general predictor in an attempt to determine how accurate it is for predicting test selection percentages for all future versions of a program. In the earlier KornShell study [23], it was determined that the relation $\operatorname{cover}_{M}(t, e)$ changes very little during maintenance. In particular, Rosenblum and Weyuker found that the coverage relation was extraordinarily stable over the 31 versions of KornShell that they included in their study: with an average of only one-third of one percent of the elements in the relation changing from version to version and only two versions for which the amount of change exceeded one percent. For this reason, Rosenblum and Weyuker argued that coverage information from a single version might be usable to guide test selection over several subsequent new versions, thereby saving the cost of redoing the coverage analysis on each new version.

However, in circumstances where the coverage relation is not stable, it may be desirable to make predictions about whether or not test selection is likely to be cost-effective for a particular version, using version-specific information. Therefore, the goal of our second study was to examine the accuracy of the RW predictor as a version-specific predictor for our subject programs, modified versions, and test suites. In some cases, the intuition is that it might be important to utilize information that is known about the specific changes made to produce a particular version.

We considered each base program $P$, with each modified version $P_{i}$ and test suite $T_{j}$, as we had done in Study 1, except that we did not compute averages over the percentages of test cases selected over all versions of a program. Instead, the data sets for this study contain one deviation for each test suite and each version of a program.

As in Study 1, the first step in our investigation was to see how much the percentage of test cases actually selected by each of the methods differed from the predicted percentage of test cases. For this analysis, we needed the following additional pieces of data, which we computed for each $P, P_{i}$, and $T_{j}$ :

$$
\begin{gathered}
D_{\text {DejaVu }_{i, j}}=\pi_{\text {DejaVu }_{j}}-S_{\text {DejaVu }_{i, j}}, \\
D_{\text {TestTube }_{i, j}}=\pi_{\text {TestTube }_{j}}-S_{\text {TestTube }_{i, j}} .
\end{gathered}
$$

$D_{\text {DejaVu }_{i, j}}$ and $D_{\text {TestTube }_{i, j}}$ represent the deviations of the percentages of the test cases predicted by the RW predictor for $T_{j}$ from the actual percentages of test cases selected by the respective method for the versions $P_{i}$ of $P$. As in Study 1, to determine the ranges into which the values for $D_{\mathrm{DejaVu}_{i, j}}$ and $D_{\text {Test Tube }_{i, j}}$ actually fell, we rounded the values of
$D_{\mathrm{DejaVu}_{i, j}}$ and $D_{\mathrm{TestTube}_{i, j}}$ to the nearest integer $I$ and computed, for each $I$ (such that $-100 \leq I \leq 100$ ), the percentage of the rounded $D$ values with value $I$. For each $P$, using each of its $D_{\mathrm{DejaVu}_{i, j}}$ values, the result was a set $H_{\text {DejaVu }}$ :

$$
\begin{gathered}
H_{\text {DejaVu }}=\{(r, p r d) \mid r \text { is the range value, }-100 \leq r \leq 100, \\
\text { prd is the percentage of rounded } \left.D_{\mathrm{DejaVu}_{i, j}} \text { values at } r\right\} .
\end{gathered}
$$

Similarly, for each $P$, using each of its $D_{\text {TestTube }_{i, j}}$ values, the result was a set $H_{\text {TestTube: }}$

$$
\begin{aligned}
& H_{\text {TestTube }}=\{(r, p r d) \mid r \text { is the range value, }-100 \leq r \leq 100, \\
& \left.\quad p r d \text { is the percentage of rounded } D_{\text {TestTube }_{i, j}} \text { values at } r\right\} .
\end{aligned}
$$

\subsection{Threats to Validity}

There are three types of potential threats to the validity of our studies: 1) threats to construct validity, which concern our measurements of the constructs of interest (i.e., the phenomena underlying the independent and dependent variables), 2) threats to internal validity, which concern our supposition of a causal relation between the phenomena underlying the independent and dependent variables, and 3) threats to external validity, which concern our ability to generalize our results.

\subsubsection{Construct Validity}

Construct validity deals directly with the issue of whether or not we are measuring what we purport to be measuring. The RW predictor relies directly on coverage information. It is true that our measurements of the coverage relation are highly accurate, but the coverage relation is certainly not the only possible phenomenon that affects the costeffectiveness of selective regression testing. Therefore, because this measure only partially captures that potential, we need to find other phenomena that we can measure for purposes of prediction.

Furthermore, we have relied exclusively on the number of test cases selected as the measure of cost reduction. Care must be taken in the counting of test cases deemed to be "selected," since there are other reasons a test case may not be selected for execution (such as the testing personnel simply lacking the time to run the test case). In addition, whereas this particular measure of cost reduction has been appropriate for the subjects we have studied, there may be other testing situations for which the expense of a test lab and testing personnel might be significant cost factors. In particular, the possibility of using spare cycles might affect the decision of whether or not it is worthwhile to use a selective regression testing method at all in order to eliminate test cases and, therefore, whether or not a predictor is meaningful.

\subsubsection{Internal Validity}

The basic premises underlying Rosenblum and Weyuker's original predictor were that 1) the cost-effectiveness of a selective regression testing method and, hence, our ability to predict cost-effectiveness, are directly dependent on the percentage of the test suite that the selective regression 
testing method chooses to run, and that 2) this percentage in turn is directly dependent on the coverage relation. In this experiment, we take the first premise as an assumption and investigate whether the relation between percentage of tests selected and coverage exists and is appropriate as a basis for prediction. The new data presented in this paper reveal that coverage explains only part of the cost-effectiveness of a method and the behavior of the RW predictor. Future studies should therefore attempt to identify the other factors that affect test selection and cost effectiveness.

\subsubsection{External Validity}

The threats to external validity of our studies are centered around the issue of how representative the subjects of our studies are. All of the subject programs in our new studies are small, and the sizes of the selected test suites are small. This means that even a selected test suite whose size differs from the average or the predicted value by one or two elements would produce a relatively large percentage difference. (Therefore, the results of Study 1 are particularly interesting because they show small average deviations for most of the subject programs.)

For the studies involving the Siemens programs, the test suites were chosen from the test pools using branch coverage, which is much finer granularity than TestTube uses, suggesting that there is a potential "mismatch" in granularity that may somehow skew the results. More generally, it is reasonable to ask whether our results are dependent upon the method by which the test pools and test suites were generated and the way in which the programs and modifications were designed. We view the branch coverage suites as being reasonable test suites that could be generated in practice, if coverage-based testing of the programs were being performed. Of course there are many other ways that testers could and do select test cases, but because the test suites we have studied are a type of suite that could be found in practice, results about predictive power with respect to such test suites are valuable.

The fact that the faults were synthetic (in the sense that they were seeded into the Siemens programs) may also affect our ability to investigate the extent to which change information can help us predict future changes. In a later section, we will introduce a new predictor that we call the weighted predictor. This predictor depends on versionspecific change information. Because it seemed likely that conclusions drawn using synthetic changes would not necessarily hold for naturally occurring faults, we did not attempt to use the Siemens programs and their faulty versions to empirically investigate the use of the weighted predictor. Nevertheless, the predictor itself is not dependent on whether the changes are seeded or naturally occurring and, thus, our results provide useful data points.

\section{Data AND Results}

\subsection{Study 1}

Fig. 1 presents data for $\bar{D}_{\mathrm{DejaVu}_{j}}$ (3) and $\bar{D}_{\text {TestTube }_{j}}$ (4), with one graph for each subject program $P$ : The Appendix gives details of the computation of this data using one of the subject programs, printtokens2, as an example.

Each graph contains a solid curve and a dashed curve. The solid curve consists of the connected set of points
$\bar{H}_{\mathrm{DejaVu}_{j}}$ (5), whereas the dashed curve consists of the connected set of points $\bar{H}_{\text {TestTube }_{j}}$ (6). Points to the left of the " 0 " deviation label on the horizontal axes represent cases in which the percentage of test cases predicted was less than the percentage of test cases selected by the tool, whereas points to the right of the " 0 " represent cases in which the percentage of test cases predicted was greater than the percentage of test cases selected by the tool. To facilitate a display of the values, a logarithmic transformation has been applied to the $y$ axes. No smoothing algorithms were applied to the curves.

For all $P$ and $T_{j}$, the $\bar{D}_{\mathrm{DejaVu}_{j}}$ were in the range of $[-20,33]$ and the $\bar{D}_{\text {Test Tube }_{j}}$ were in the range of $[-24,28]$. However, as we shall see in Fig. 2, these ranges are a bit misleading because there are rarely any significant number of values outside the range of $(-10,0]$ or $[0,10)$, particularly for Test Tube.

The graphs show that, for our subjects, the RW predictor was quite successful for both the DejaVu and TestTube selection methods. The predictor was least successful for the printtokens 2 program for which it predicted an average of 23 percent more test cases than DejaVu actually selected. This was the only deviation that exceeded 10 percent using the DejaVu approach. For schedule1, the prediction was roughly 9 percent higher, on average, compared to the DejaVu-selected test suite. DejaVu selected an average of roughly 10 percent more test cases than predicted for schedule2, 7 percent more for totinfo, 7 percent more for tcas, 3 percent more for printtokens1, and 4 percent fewer for replace than the RW predictor predicted.

For TestTube, the predictor also almost always predicted within 10 percent of the actual average number of test cases that were actually selected. The only exception was for the totinfo program, for which the average deviation was under 12 percent. For the other programs, the average deviations were 5 percent for the printtokens 1 program, 5 percent for the printtokens 2 program, 4 percent for the replace program, 7 percent for the tcas program, 10 percent for schedule1, and 1 percent for schedule2. We consider these results encouraging, although not as successful as the results described by Rosenblum and Weyuker for the KornShell case study. Recall that, in that study, there were a total of 31 versions of KornShell, a large program with a very large user base, with all changes made to fix real faults or modify functionality. None of the changes were made for the purpose of the study.

Another way to view the data for this study is to consider deviations of the predicted percentage from the actual percentage without considering whether the predicted percentage was greater or less than the actual percentage selected. These deviations constitute the absolute value deviation. To compute the absolute value deviation, we performed some additional computations:

For each $P$ and each $T_{j}$, we first computed $A b s \bar{D}_{\text {DejaVu }_{j}}=$ $\left|\bar{D}_{\text {DejaVu }_{j}}\right|$ and $A b s \bar{D}_{\text {TestTube }_{j}}=\left|\bar{D}_{\text {TestTube }_{j}}\right|$. We then tabulated the percentage of the $A b s \bar{D}_{\mathrm{DejaVu}_{j}}$ and the $A b s \bar{D}_{\text {TestTube }_{j}}$ that fell in each of the ranges [0 percent, 10 percent], [10 percent, 20 percent], . . ., [90 percent, 100 percent].

Fig. 2 depicts these results as segmented bar graphs. The figure contains two bars for each subject program: The left bar of each pair represents the absolute value deviation of DejaVu results from the RW predictor, and the right bar represents the absolute value deviation of TestTube 

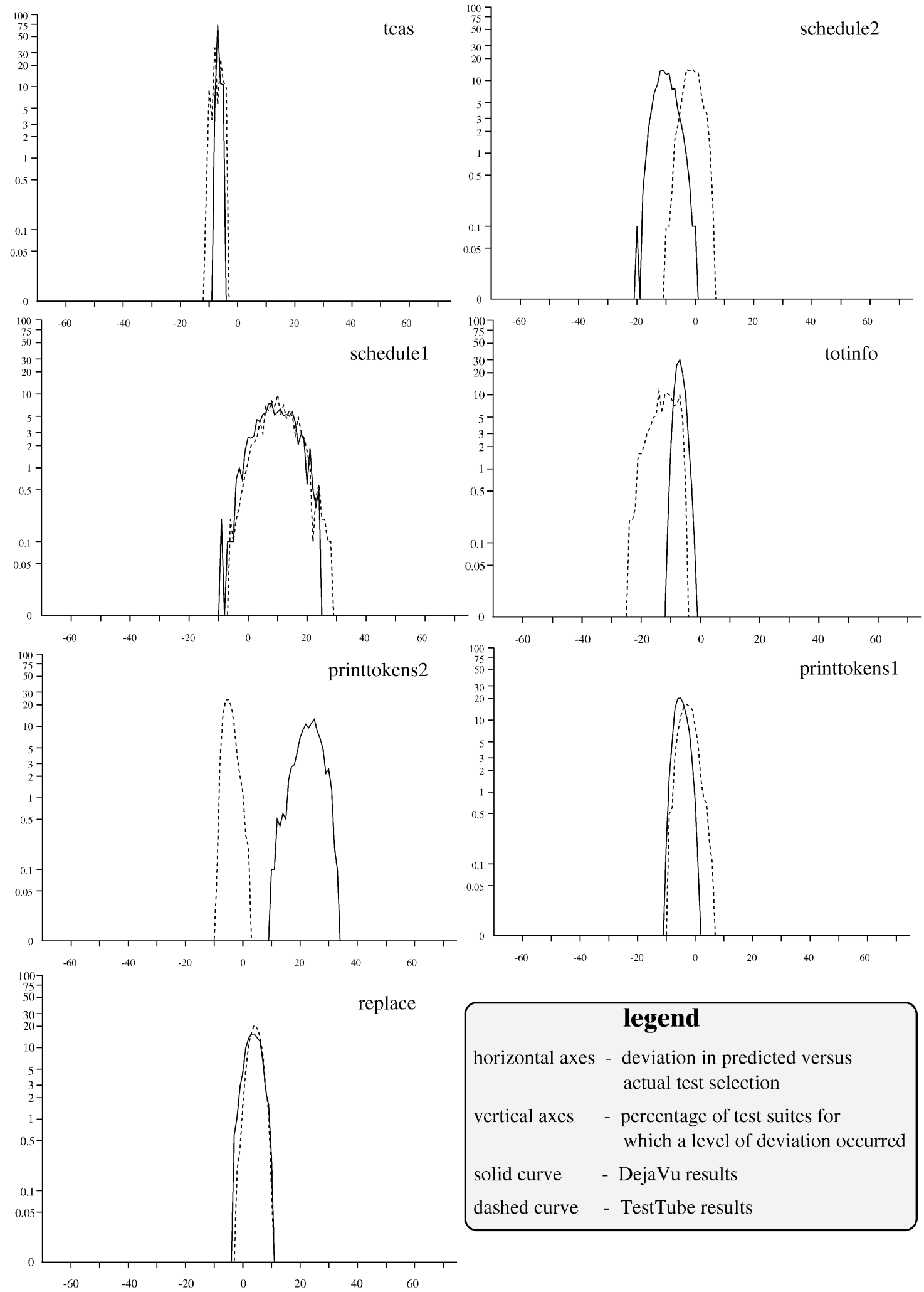

Fig. 1. Deviation between predicted and actual test selection percentages for application of DejaVu and TestTube to the subject programs. The figure contains one graph for each subject program. In each graph, the solid curve represents deviations for DejaVu, and the dashed curve represents deviations for Test Tube.

results from the RW predictor. For each program $P$, these numbers are averages over all versions $P_{i}$ of $P$. Each bar represents 100 percent of the test suites $T_{j}$, with shading used to indicate the percentage of test suites whose deviations fell within the corresponding range. For instance, in the case of printtokens 2,100 percent of the test suites showed less than 10 percent deviation for TestTube, whereas for DejaVu, 14 percent of the test suites showed deviations between 10 percent and 20 percent, 82 percent showed deviations between 20 percent and 30 percent, and 


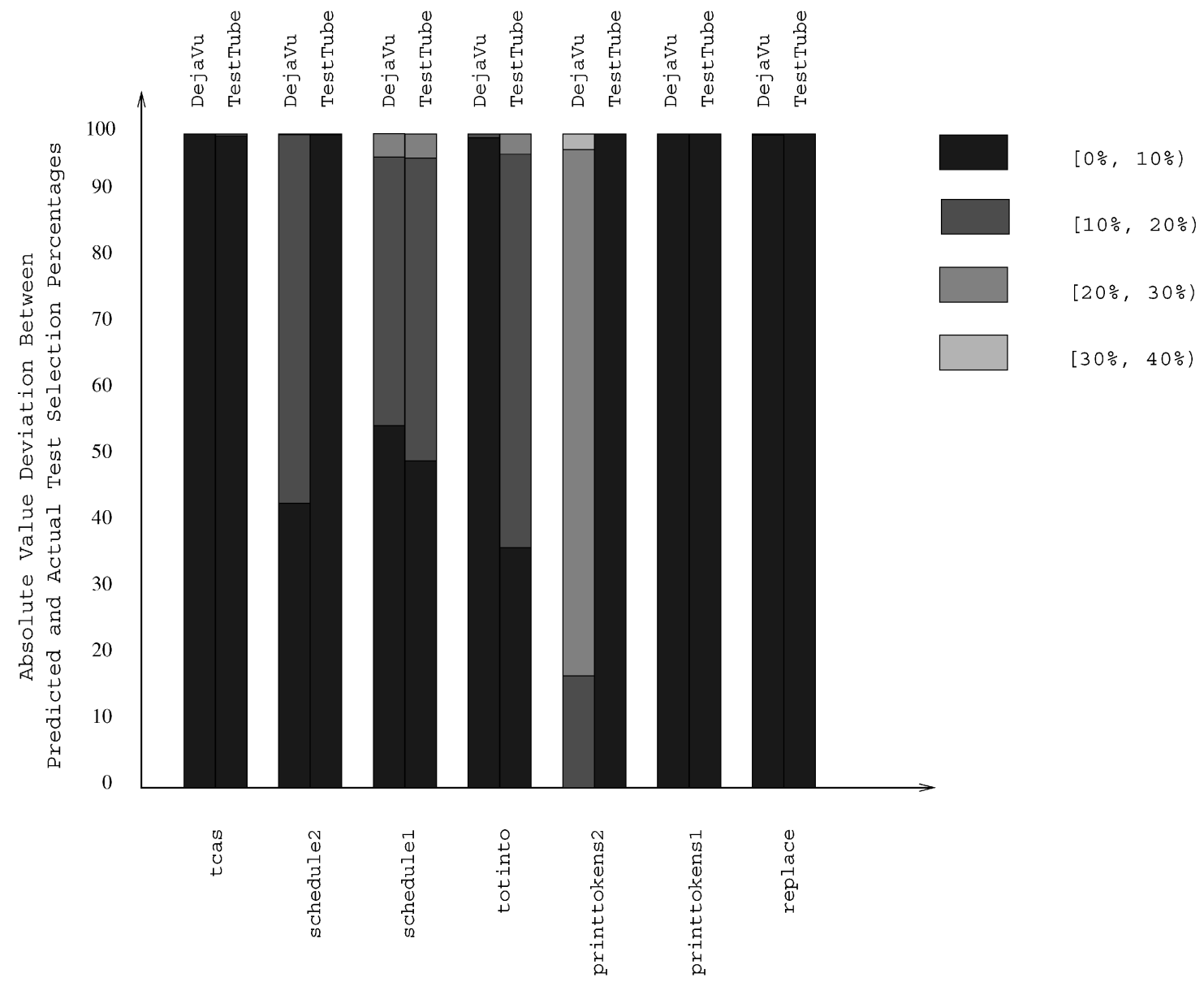

Fig. 2. Absolute value deviation between predicted and actual test selection percentages for application of DejaVu and TestTube to the subject programs. The figure contains two bars for each subject program: the left bar of each pair represents the absolute value deviation of DejaVu results from the RW predictor and the right bar represents the absolute value deviation of Test Tube results from the RW predictor. For each program $P$, these numbers are averages over all versions $P_{i}$ of $P$. Each bar represents 100 percent of the test suites $T_{j}$, with shading used to indicate the percentage of test suites whose deviations fell within the corresponding range.

4 percent showed deviations between 30 percent and 40 percent.

The results of this study show that, for many of the subject programs, modified versions, and test suites, the absolute value deviation for both DejaVu and TestTube was less than 10 percent. In these cases, the RW model explains a significant portion of the data. However, in a few cases, the absolute value deviation was significant. For example, as mentioned above, for printtokens2, the absolute value deviation from the predictor for DejaVu was between 20 percent and 30 percent for more than 80 percent of the versions.

One additional feature of the data displayed in Fig. 1 bears discussion. For all programs other than printtokens2, the curves that represent deviations for DejaVu and TestTube are (relatively) close to one another. For printtokens 2 , in contrast, the two curves are disjoint and (relatively) widely separated. Examination of the code coverage data and locations of modifications for the programs reveals reasons for this difference.

Sixteen of the nineteen printtokens 2 functions are executed by a large percentage (on average over 95 percent) of the test cases in the program's test pool; the remaining three functions are executed by much lower percentages (between 20 percent and 50 percent) of the test cases in that test pool. All modifications of printtokens 2 occur in the sixteen functions that are executed by nearly all test cases. Thus, the actual test selections by TestTube, on average, include most test cases. The presence of the latter three functions, and the small number of test cases that reach them, however, causes a reduction in the average number of test cases per function, and causes the function-level predictor to under-predict by between 0 percent and 10 percent the number of test cases selected by TestTube.

Even though nearly all test cases enter nearly all functions in printtokens2, several of these functions contain branches that significantly partition the paths taken by test cases that enter the functions. Thus, many of the statements in printtokens 2 are actually executed by fewer than 50 percent of the test cases that enter their enclosing functions. When modifications occur in these lessfrequently executed statements, DejaVu selects much smaller test suites than TestTube. (For further empirical comparison of TestTube and DejaVu, see [22].) This is the case for approximately half of the modified versions of printtokens 2 utilized in this study. However, the presence of a large number of statements that are executed by a larger proportion of the test cases causes the average number of test cases per statement to exceed the number of test cases through modified statements. The end result is 
that the statement-level predictor overpredicts the number of test cases selected by DejaVu by between 5 percent and 27 percent. Of course, the precise locations of modifications in the subjects directly affect the results. Therefore, the fact that all changes were synthetic is of concern when trying to generalize these results.

\subsection{Study 2}

Like Fig. 1, Fig. 3 contains one graph for each subject program. The graphs also use the same notation as was used in Fig. 1, using a solid curve to represent the percentage of occurrences of $D_{\mathrm{DejaVu}_{i, j}}$ over deviations for all test suites $T_{j}$ and using a dashed curve to represent the percentage of occurrences of $D_{\text {TestTube }_{i, j}}$ over deviations for all test suites $T_{j}$. Again, a logarithmic transformation has been applied to the y axes. Fig. 4 depicts these results as a segmented bar graph, in the manner of Fig. 2.

The results of this study show that, for the subject programs, modified versions, and test cases, the deviations and absolute value deviations for individual versions for both DejaVu and TestTube are much greater than in Study 1 . This is not surprising because in this study, the results are not averaged over all versions as they were in Study 1. For example, consider tcas, printtokens 1 , and replace. In Study 1, the average absolute value deviation from the predicted percentage for each of these programs is less than 10 percent using either DejaVu or TestTube. However, when individual versions are considered, the percentage of test cases selected by DejaVu for these programs varies significantly, up to 64 percent, from the percentages predicted. Deviations and absolute value deviations for the other subjects show similar differences. In Fig. 3, the range of deviations can be seen. In most cases, there are at least a few versions that have a small number of instances for which the deviations are significant. The bar graphs in Fig. 4 show more clearly how frequently these large absolute value deviations occur.

In Fig. 3, the data for printtokens2 is again particularly interesting. In this case, the curve for TestTube is peaked and relatively narrow, whereas the curve for DejaVu is nearly flat and relatively wide. As discussed in the preceding section, for both techniques, these differences reflect differences in the degree of variance in the coverage relations at the statement and function level, as well as differences in the location of modifications. In this case, however, considering prediction on a version-specific basis causes the deviation in prediction at the statement level, where the variance in coverage is large, to be flat. Lack of variance in coverage at the function level prevents the Test Tube curve from being flat.

\section{Improved Predictors}

As discussed in Section 3.4, the assumptions underlying our measurement of costs may pose threats to the validity of our conclusions about predictions of cost-effectiveness using the RW predictor. Furthermore, in some of the subject programs of our studies, there was significant absolute deviation of the results of the selective regression testing tools (DejaVu and TestTube) with respect to test selection values predicted by the RW predictor. Therefore, we believe that there may be factors affecting cost-effectiveness that are not being captured by the RW predictor. These factors, if added to the model, could improve the accuracy of both general and version-specific predictors. The RW predictor accounts for test coverage but does not account for the locations of modifications. Therefore, one obvious refinement would be to incorporate information about modifications into the predictor. We saw in Study 2 that the specific changes made to create a particular version may have significant effects on the accuracy of prediction in practice. Thus, we believe that an extended weighted predictor might be more accurate for both general and version-specific prediction. Such a predictor would incorporate information about the locations of the changes and weight the predictor accordingly.

To this end, in this section, we extend the RW predictor by adding weights that represent the relative frequency of changes to the covered entities. For each element $e_{j} \in E^{\mathcal{C}}, w_{j}$ is the relative frequency with which $e_{j}$ is modified, and it is defined such that $\sum_{j=1}^{\left|E^{\mathcal{C}}\right|} w_{j}=1$. The original, unweighted RW model, discussed in Section 2, computes the expected number of test cases that would be rerun if a single change is made to a program. To do this, the model uses the average number of test cases that cover each covered entity $e_{j} \in E^{\mathcal{C}}$. This average is referred to as $N_{M}^{\mathcal{C}}$. The weighted analogue of $N_{M}^{\mathcal{C}}$ is a weighted average, $W N_{M}^{\mathcal{C}}$, which we define as follows:

$$
W N_{M}^{\mathcal{C}}=\sum_{j=1}^{\left|E^{\mathcal{C}}\right|} w_{j} \sum_{i=1}^{|T|} C_{i, j},
$$

where $C_{i, j}$ is defined as before:

$$
C_{i, j}= \begin{cases}1 & \text { if } \operatorname{covers}_{M}(i, j) \\ 0 & \text { otherwise }\end{cases}
$$

Note that the inner sum represents the total number of test cases covered by $e_{j}$; multiplying that sum by $w_{j}$ provides $e_{j}$ 's weighted contribution to the total number of test cases selected overall.

For this weighted average, the fraction of the test suite $T$ that must be rerun, denoted by $\Pi_{M}$, is given as follows:

$$
\Pi_{M}=\frac{W N_{M}^{\mathcal{C}}}{|T|} .
$$

Note that the original, unweighted RW predictor, $\pi_{M}$, is a version of this weighted predictor in which $w_{j}$ is $\frac{1}{\left|E^{\mathcal{C}}\right|}$ for all $e_{j}$ (which represents an assumption that each entity is equally likely to be changed):

$$
\begin{aligned}
\Pi_{M} & =\frac{W N_{M}^{\mathcal{C}}}{|T|} \\
& =\frac{\sum_{j=1}^{\left|E^{\mathcal{C}}\right|} \frac{1}{\left|E^{\mathcal{C}}\right|} \sum_{i=1}^{|T|} C_{i, j}}{|T|} \\
& =\frac{\sum_{j=1}^{\left|E^{\mathcal{C}}\right|} \sum_{i=1}^{|T|} C_{i, j}}{\left|E^{\mathcal{C}}\right||T|} \\
& =\frac{C C}{\left|E^{\mathcal{C}}\right||T|} \\
& =\pi_{M} .
\end{aligned}
$$



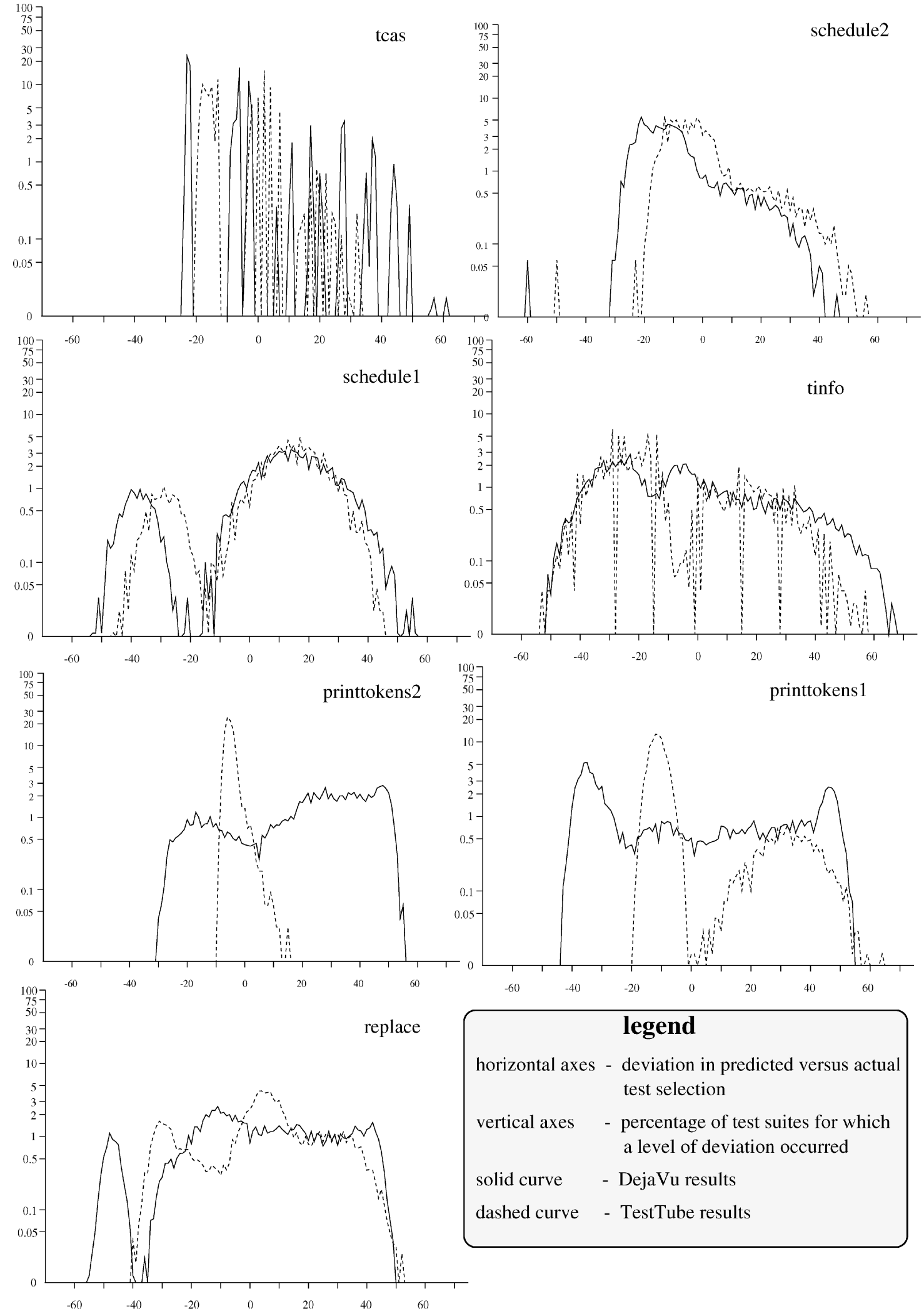

Fig. 3. Version-specific absolute value deviation between predicted and actual test selection percentages for application of DejaVu and TestTube to the subject programs. The figure contains one graph for each subject program. In each graph, the solid curve represents deviations for DejaVu and the dashed curve represents deviations for TestTube.

To see the impact of the difference between $\pi_{M}$ and $\Pi_{M}$, consider coverage patterns $A$ and $B$, shown respectively in Figs. 5 and 6, where each dot represents an entity and each closed curve a test case. Assume that the patterns are generalized over a large number of entities, $n$. As discussed by Rosenblum and Weyuker [23], the value of $\pi_{M}$ predicted 


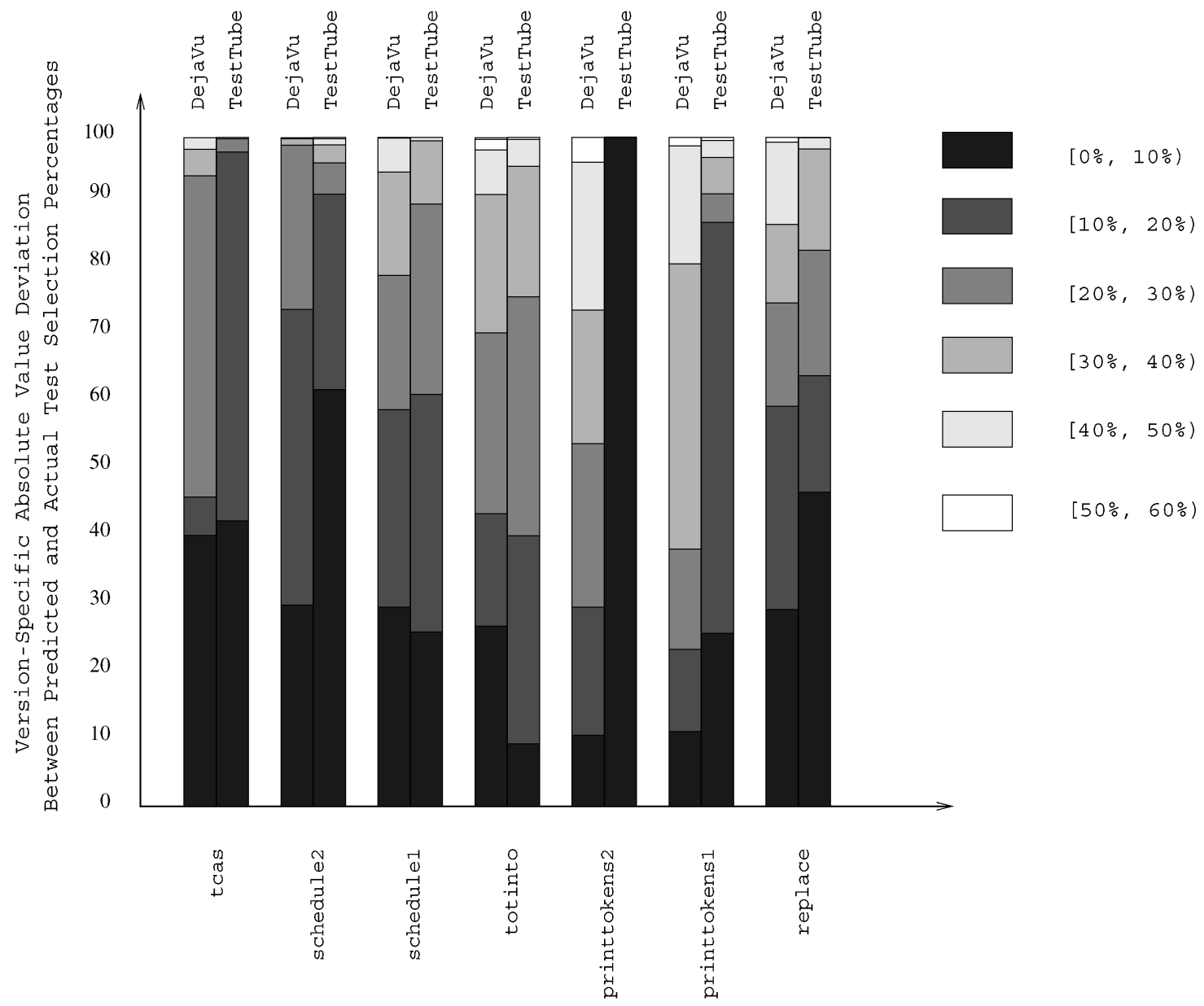

Fig. 4. Version-specific absolute value deviation between predicted and actual test selection percentages for application of DejaVu and TestTube to the subject programs. The figure contains two bars for each subject program: The left bar of each pair represents the absolute value deviation of DejaVu results from the RW predictor and the right bar represents the absolute value deviation of TestTube results from the RW predictor. Each bar represents 100 percent of the test suites $T_{j}$, with shading used to indicate the percentage of test suites whose deviations fell within the corresponding range.

for each pattern is $2 / n$. In Pattern $A$, the test cases are distributed evenly over the entities and, thus, $\Pi_{M}$ and $\pi_{M}$ are the same and yield the exact number of test cases that would be selected by either DejaVu or TestTube, regardless of the relative frequency of changes (and, hence, regardless of the values assigned to the $w_{j}$ ).

In Pattern $B$, the test cases are not distributed evenly over the entities and, in contrast with Pattern $A$, the RW predictor never predicts the exact fraction selected for any changed entity, and it is significantly inaccurate for a change to the "core" element of that pattern. Suppose, however, that instead of assuming that the frequency of change is equal for all entities, we had information about the relative frequency of modifications to individual entities. In this case, using the weighted predictor, we could compute a more accurate estimate of the fraction of the test suite that would be selected. For example, if we knew that changes are always made to two of the noncore entities (with one changed exactly as often as the other) and that no other entities are ever changed, then the weights would be $1 / 2$ for the two changed entities and 0 for all other entities. And, thus, we would predict that (for the case of a single entity change) $1 / n$ of the test suite would be selected, rather than $2 / n$ as predicted by the unweighted predictor.

\subsection{Improved General Prediction}

Provided we can obtain values for weights that accurately model the distribution of future modifications to a program, we can use the weighted predictor, $\Pi_{M}$, to improve general prediction. One approach is to utilize change history information about the program, often available from configuration management systems. Assuming that the change histories do accurately model the pattern of future modifications (a result suggested by the work of Harrison and Cook [11]), we can use this information to compute weights for $\Pi_{M}$. If the change histories are recorded at the module level, $\Pi_{M}$ can also be used to predict the percentage of test cases selected on average by a tool, such as TestTube, that considers module-level changes to the system. If the change histories are recorded at the statement level, $\Pi_{M}$ can be used to predict the percentage of test cases selected on average by a tool, such as DejaVu, that considers statement-level changes to the system. In either case, the weighted predictor can be used to incorporate data that may account for change-location information, without performing full change analysis. Thus, it can be used to assess whether it will be worthwhile to perform all of the analysis needed by a selective regression testing tool. 


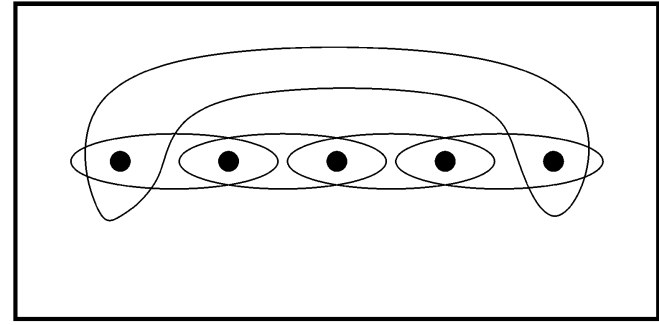

Fig. 5. Coverage pattern A.

In practice, weights may be collected and assumed to be fixed over a number of subsequent versions of a program, or they may be adjusted as change history information becomes available. In this context, an important consideration involves the extent to which weights collected at a particular time in the history of a program can continue to predict values for future versions of that program and the extent to which the accuracy of predictions based on those weights may decrease over time. Future empirical study of this issue is necessary.

\subsection{Improved Version-Specific Prediction}

We can also use the weighted predictor, $\Pi_{M}$, as a versionspecific predictor. For this version-specific predictor, one approach computes the $w_{i}$ using the configuration management system. We assign a weight of $1 / k$ to each entity that has been changed (where $k$ is the total number of entities changed), and we assign a weight of 0 to all other entities in the system. Using these weights, $\Pi_{M}$ computes the exact percentage of test cases that will be selected by a test selection tool that selects at the granularity of the entities. For example, if the entities are modules, then $\Pi_{M}$ will predict the exact percentage of test cases that will be selected by a test selection tool, such as TestTube, that considers changes at the module level. If the entities are statements, then $\Pi_{M}$ will predict the exact percentage of test cases that will be selected by a test selection tool, such as DejaVu, that considers changes at the statement level. If the cost of determining the number of test cases that will be selected is cheaper than the cost of actually selecting the test cases, this approach can be cost-effective.

It is worth noting that Rosenblum and Weyuker found, in their experiments with KornShell, that it was typically not necessary to recompute the coverage relation frequently, because it remained very stable over the 31 versions they studied. If this is typical of the system under test, then this should make version-specific predictors extremely efficient to use and, therefore, provide valuable information about whether or not the use of a selective regression testing strategy is likely to be cost-effective for the current version of the system under test.

An alternative approach assumes that method $M$ can be supplemented with an additional change analysis capability that is more efficient but less precise than $M^{\prime}$ 's change analysis. This supplementary change analysis is used during the critical phase of regression testing-after all modifications have been made to create $P^{\prime}$, the new version

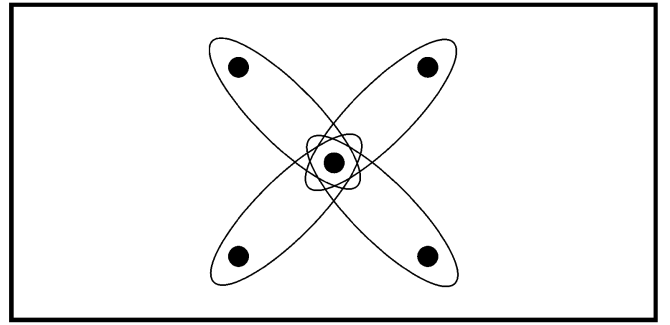

Fig. 6. Coverage pattern B.

of $P .^{3}$ The results of the supplementary change analysis can be used to assign weights to the entities in the system, which are then used for prediction as described above.

Using the weighted predictor, $\Pi_{M}$, as a version-specific predictor will be especially appropriate for test suites whose test cases are not evenly distributed across the entities, such as the case illustrated by Pattern $B$, where test selection results for specific versions may differ widely from average test selection results over a sequence of versions.

\section{Conclusions}

In this paper, we presented results from new empirical studies that were designed to evaluate the effectiveness and accuracy of the Rosenblum-Weyuker (RW) model for predicting cost-effectiveness of a selective regression testing method. The RW model was originally framed solely in terms of code coverage information and evaluated empirically using the TestTube method and a sequence of 31 versions of KornShell. In the new studies, two selective regression testing methods were used (TestTube and DejaVu), and seven different programs were used as subjects. For the experimental subjects we used in the new studies, the original RW model frequently predicted the average overall effectiveness of the two test selection techniques with an accuracy that we believe is acceptable given that the cost assumptions underlying the RW model are quite realistic for our subjects. However, the predictions of the model occasionally deviated significantly from observed test selection results. Moreover, when this model was applied to the problem of predicting test selection results for particular modified versions of the subject programs, its predictive power decreased substantially, particularly for DejaVu. These results suggest that the distribution of modifications made to a program can play a significant role in determining the accuracy of a predictive model of test selection. Therefore, we conclude that to achieve improved accuracy both in general and when applied in a version-specific manner, prediction models must account for both code coverage and modification distribution.

In response to this result, we showed how to extend the Rosenblum-Weyuker predictor to incorporate information

3. Rothermel and Harrold divide regression testing into two phases for the purpose of cost analysis. During the preliminary phase, changes are made to the software, and the new version of the software is built. During the critical phase, the new version of the software is tested prior to its release to customers [25]. 
TABLE 2

Partial Data Used in the Computation of Graphs in Fig. 1

\begin{tabular}{|c|rrrrrrrrr|}
\hline & \multicolumn{10}{|c|}{ Test Suites } \\
\cline { 2 - 10 } & $T_{1}$ & $T_{2}$ & $T_{3}$ & $T_{4}$ & $T_{5}$ & $T_{6}$ & $T_{7}$ & $T_{8-999}$ & $T_{1000}$ \\
\hline \hline$\pi_{\text {DejaVu }_{j}}$ & 54.8 & 61.6 & 53.0 & 54.0 & 58.0 & 55.1 & 56.0 & $\ldots$ & 54.8 \\
\hline \hline$S_{\text {DejaVu }_{i, j}}$ & \multicolumn{10}{|c}{} & & & & & & & \\
\hline Version 1 & 58.3 & 81.8 & 62.5 & 60.0 & 84.6 & 62.5 & 72.7 & $\ldots$ & 50.0 \\
\hline Version 2 & 8.3 & 9.1 & 12.5 & 10.0 & 7.7 & 12.5 & 9.1 & $\ldots$ & 7.1 \\
\hline Version 3 & 16.7 & 18.2 & 25.0 & 10.0 & 23.1 & 12.5 & 18.2 & $\ldots$ & 21.4 \\
\hline Version 4 & 41.7 & 18.2 & 25.0 & 30.0 & 38.5 & 37.5 & 36.4 & $\ldots$ & 35.7 \\
\hline Version 5 & 8.3 & 9.1 & 12.5 & 10.0 & 7.7 & 12.5 & 9.1 & $\ldots$ & 7.1 \\
\hline Version 6 & 16.7 & 45.5 & 25.0 & 20.0 & 30.8 & 25.0 & 18.2 & $\ldots$ & 14.3 \\
\hline Version 7 & 41.7 & 18.2 & 25.0 & 30.0 & 38.5 & 37.5 & 36.4 & $\ldots$ & 35.7 \\
\hline Version 8 & 66.7 & 81.8 & 62.5 & 70.0 & 76.9 & 75.0 & 81.8 & $\ldots$ & 71.4 \\
\hline Version 9 & 41.7 & 18.2 & 25.0 & 30.0 & 38.5 & 37.5 & 36.4 & $\ldots$ & 35.7 \\
\hline Version 10 & 33.3 & 54.5 & 50.0 & 40.0 & 23.1 & 50.0 & 36.4 & $\ldots$ & 28.6 \\
\hline \hline $\bar{S}_{\text {Dejavu }_{j}}$ & 33.3 & 35.5 & 32.5 & 31.0 & 36.9 & 36.2 & 35.5 & $\ldots$ & 30.7 \\
\hline \hline $\bar{D}_{\text {Dejavu }_{j}}$ & 21.5 & 26.1 & 20.5 & 23.0 & 21.1 & 18.9 & 20.5 & $\ldots$ & 24.1 \\
\hline \hline
\end{tabular}

on the distribution of modifications. However, to judge the efficacy of this extended predictive model in practice, we require additional experimentation. For this purpose, the subjects used in the studies reported in this paper will not suffice. Rather, we require versions of a program that form a succession of changes over their base versions, as the versions of KornShell did. We are currently building a repository of such programs and versions that, when complete, will provide subjects suitable for further empirical investigation of predictive models for regression testing in general and of our weighted predictor in particular.

Future studies must be directed not only toward further validation of the RW predictor and the improved predictors described in this paper, but toward the development of a more realistic cost model for regression testing. This will require extensive field studies of existing large systems in order to create a better picture of the different factors driving cost-effectiveness, such as test suite size, test case execution times, testing personnel costs, and the availability of spare machine cycles for regression testing.

\section{APPENDIX}

\section{Detalls of the Computation OF DATA FOR FIGS. 1 AND 3}

To compute the data used for the graphs in Fig. 1, we used a procedure described in Section 3.3.1. As further explanation, we give details of the computation of that data for one subject program, printtokens 2 .

For our experiments, we used 1,000 coverage-based test suites, $T_{1}, \ldots T_{1000}$. Table 2 shows data for a subset of these test suites: $T_{1}-T_{7}$ and $T_{1000}$. For each test suite $T_{j}$, we used the RW predictor to predict the number of test cases that would be selected by DejaVu when an arbitrary change is made to printtokens2. We then used this number to determine $\pi_{\mathrm{DejaVu}_{j}}$, the percentage of test cases that the RW predictor predicts will be selected by DejaVu when an arbitrary change is made to printtokens 2 . The first row of Table 2 gives these percentages for $T_{1}-T_{7}$ and $T_{1000}$.

We had ten versions of printtokens2 (see Table 1). Next, we ran DejaVu on these ten versions, with each of the 1,000 test suites and, for each version $i$ and test suite $j$, recorded the number of test cases selected. We then used this number to compute, for each $i$ and $j, S_{\mathrm{DejaVu}_{i, j}}$, the percentage of test cases selected. The ten rows for Versions 1-10 in Table 2 give these percentages. For example, from the table, we can see that, for $T_{1}, S_{\mathrm{DejaVu}_{i, 1}}$ ranges from 8.3 percent to 66.7 percent. Using (1), we then computed the $\bar{S}_{\mathrm{DejaVu}_{j}}$ for each test suite $T_{j}$. Table 2 gives these percentages for each $T_{j}$.

We then used (3) to compute, for each $T_{j}$, the difference between the percentage predicted by the RW predictor and the average percentage selected by DejaVu (i.e., $\bar{D}_{\mathrm{DejaVu}_{j}}$ ). Table 2 shows that, for $T_{1}-T_{7}$ and $T_{1000}$, the $\bar{D}_{\mathrm{DejaVu}_{j}}$ range from 18.9 percent to 26.1 percent.

Finally, we created the set, $\bar{H}_{\mathrm{DejaVu}}$ (5). The ordered pairs in this set are obtained by first rounding the percentages of the $\bar{D}_{\mathrm{DejaVu}_{j}}$, then determining the number of those rounded percentages that have range value $-100 \leq r \leq 100$, and then determining the percentage of those percentages that occur for each value of $r$. Thus, for printtokens2, $\bar{D}_{\mathrm{DejaVu}_{6}}$ rounds to $19, \bar{D}_{\mathrm{DejaVu}_{3}}, \bar{D}_{\mathrm{DejaVu}_{5}}$, and $\bar{D}_{\mathrm{DejaVu}_{7}}$ round to 21, $\bar{D}_{\mathrm{DejaVu}_{1}}$ rounds to $22, \bar{D}_{\mathrm{DejaVu}_{4}}$ rounds to $23, \bar{D}_{\mathrm{DejaVu}_{1000}}$ rounds to 24 , and $\bar{D}_{\mathrm{DejaVu}_{2}}$ rounds to 26 . Thus, there will be ordered pairs in $\bar{H}_{\text {DejaVu }}$ with first coordinates $19,21,22,21$, 24 , and 26, and the number of rounded percentages for $T_{1} \ldots T_{1000}$ are used to compute the percentage of times (among the 1,000 test suites) each percentage occurs, which is then used in the computation of the second coordinates of the ordered pairs. We used these ordered pairs to plot the solid curve for printtokens 2 in Fig. 1.

We used a similar approach to obtain the data for Fig. 3 except that we did not compute the averages of the deviations. To compute the data used for the graphs in 
TABLE 3

Partial Data Used in the Computation of Graphs in Fig. 3

\begin{tabular}{|c|c|c|c|c|c|c|c|c|c|}
\hline & \multicolumn{9}{|c|}{ Test Suites } \\
\hline & $T_{1}$ & $T_{2}$ & $T_{3}$ & $T_{4}$ & $T_{5}$ & $T_{6}$ & $T_{7}$ & $T_{8-999}$ & $T_{1000}$ \\
\hline$\pi_{\text {DejaVu }_{j}}$ & 54.8 & 61.6 & 53.0 & 54.0 & 58.0 & 55.1 & 56.0 & $\ldots$ & 54.8 \\
\hline \multicolumn{10}{|l|}{$S_{\text {DejaVu }_{i, j}}$} \\
\hline Version 1 & 58.3 & 81.8 & 62.5 & 60.0 & 84.6 & 62.5 & 72.7 & $\ldots$ & 50.0 \\
\hline Version 2 & 8.3 & 9.1 & 12.5 & 10.0 & 7.7 & 12.5 & 9.1 & $\ldots$ & 7.1 \\
\hline Version 3 & 16.7 & 18.2 & 25.0 & 10.0 & 23.1 & 12.5 & 18.2 & $\ldots$ & 21.4 \\
\hline Version 4 & 41.7 & 18.2 & 25.0 & 30.0 & 38.5 & 37.5 & 36.4 & $\ldots$ & 35.7 \\
\hline Version 5 & 8.3 & 9.1 & 12.5 & 10.0 & 7.7 & 12.5 & 9.1 & $\ldots$ & 7.1 \\
\hline Version 6 & 16.7 & 45.5 & 25.0 & 20.0 & 30.8 & 25.0 & 18.2 & $\ldots$ & 14.3 \\
\hline Version 7 & 41.7 & 18.2 & 25.0 & 30.0 & 38.5 & 37.5 & 36.4 & $\ldots$ & 35.7 \\
\hline Version 8 & 66.7 & 81.8 & 62.5 & 70.0 & 76.9 & 75.0 & 81.8 & $\ldots$ & 71.4 \\
\hline Version 9 & 41.7 & 18.2 & 25.0 & 30.0 & 38.5 & 37.5 & 36.4 & $\ldots$ & 35.7 \\
\hline Version 10 & 33.3 & 54.5 & 50.0 & 40.0 & 23.1 & 50.0 & 36.4 & $\ldots$ & 28.6 \\
\hline \multicolumn{10}{|l|}{$D_{\text {DejaVu }_{i, j}}$} \\
\hline Version 1 & -3.5 & -20.2 & -9.5 & -6.0 & -26.6 & -7.4 & -16.9 & $\ldots$ & 4.8 \\
\hline Version 2 & 46.5 & 52.5 & 40.5 & 44.0 & 50.3 & 42.6 & 46.9 & $\ldots$ & 47.7 \\
\hline Version 3 & 38.1 & 43.4 & 28.0 & 44.0 & 34.9 & 42.6 & 37.8 & $\ldots$ & 33.4 \\
\hline Version 4 & 13.1 & 43.4 & 28.0 & 24.0 & 19.5 & 17.6 & 19.6 & $\ldots$ & 19.1 \\
\hline Version 5 & 46.5 & 52.5 & 40.5 & 44.0 & 50.3 & 42.6 & 46.9 & $\ldots$ & 47.7 \\
\hline Version 6 & 38.1 & 16.1 & 28.0 & 34.0 & 27.2 & 30.1 & 37.8 & $\ldots$ & 40.5 \\
\hline Version 7 & 13.1 & 43.4 & 28.0 & 24.0 & 19.5 & 17.6 & 19.6 & $\ldots$ & 19.1 \\
\hline Version 8 & -11.9 & -20.2 & -9.5 & -16.0 & -18.9 & -19.9 & -25.8 & $\ldots$ & -16.6 \\
\hline Version 9 & 13.1 & 43.4 & 28.0 & 24.0 & 19.5 & 17.6 & 19.6 & $\ldots$ & 19.1 \\
\hline Version 10 & 21.5 & 7.1 & 3.0 & 14.0 & 34.9 & 5.1 & 19.6 & $\ldots$ & 26.2 \\
\hline
\end{tabular}

Fig. 3, we used a procedure described in Section 3.3.2. As further explanation, we give details of the computation of that data for one subject program, printtokens 2 .

Table 3 shows data for a subset of the 1,000 coveragebased test suites: $T_{1}-T_{7}$ and $T_{1000}$. For each test suite $T_{i}$, we used the RW predictor to predict the number of test cases that would be selected by DejaVu when an arbitrary change is made to printtokens2. We then used this number to determine $\pi_{\mathrm{DejaVu}_{j}}$, the percentage of test cases that the RW predictor predicts will be selected by DejaVu when an arbitrary change is made to printtokens2. The first row of Table 3 gives these percentages for $T_{1}-T_{7}$ and $T_{1000}$.

Next, we ran DejaVu on the ten versions of printtokens 2 and, for each version, recorded the number of test cases selected. We then used this number to compute $S_{\mathrm{DejaVu}_{i, j}}, j=1 \ldots 10$, the percentage of test cases selected. The ten rows for Versions 1-10 in Table 3 give these percentages.

We then used (7) to compute, for each version $i$ and each $T_{j}, D_{\mathrm{DejaVu}_{i, j}}$, the difference between the percentage predicted by the RW predictor and the percentage selected by DejaVu. Table 3 shows that, for $T_{1}-T_{7}$ and $T_{1000}$, these percentages range from -26.6 percent to 52.5 percent.

Finally, we created the set, $H_{\text {DejaVu }}$ (9). These ordered pairs are obtained by first rounding the percentages of the $D_{\mathrm{DejaVu}_{i, j}}$, determining the number of those rounded percentages that have range value $-100 \leq r \leq 100$, and then determining the percentage of those percentages that occur for each value of $r$. For example, for printtokens2,
$D_{\mathrm{DejaVu}_{1,2}}, \quad D_{\mathrm{DejaVu}_{8,2}}$, and $D_{\mathrm{DejaVu}_{8,6}}$ round to -20 and $D_{\mathrm{DejaVu}_{4,5}}, D_{\mathrm{DejaVu}_{7,5}}, D_{\mathrm{DejaVu}_{9,5}}, D_{\mathrm{DejaVu}_{4,7}}, D_{\mathrm{DejaVu}_{7,7}}, D_{\text {DejaVu }_{9,7}}$, and $D_{\mathrm{DejaVu}_{10,7}}$ round to 20 . Thus, $H_{\mathrm{DejaVu}}$ contains ordered pairs with -20 and 20 as the first coordinates, and the number of rounded percentages for $T_{1} \ldots T_{1000}$ are used to compute the percentage of times (among the 1,000*10 testsuite/version pairs) each percentage occurs and is used in the computation of the second coordinates of the ordered pairs.

We used this procedure to obtain the data for the rest of the graphs in Fig. 3 for DejaVu and used a similar procedure to obtain the data for the graphs for TestTube.

\section{ACKNOWLEDGMENTS}

This work was supported in part by a grant from Microsoft, Inc., by the US National Science Foundation under NYI award CCR-9696157 to Ohio State University, the CAREER award CCR-9703108 to Oregon State University, the Experimental Software Systems award CCR-9707792 to Ohio State University and Oregon State University, and by an Ohio State University Research Foundation Seed Grant. The authors thank Huiyu Wang who performed some of the experiments, Panickos Palletas and Qiang Wang who advised us on the statistical analysis, Jim Jones who performed some of the statistical analysis, and Monica Hutchins and Tom Ostrand of Siemens Corporate Research for supplying the subject programs and other data necessary for the experimentation. 


\section{REFERENCES}

[1] H. Agrawal, J. Horgan, E. Krauser, and S. London, "Incremental Regression Testing," Proc. Conf. Software Maintenance-1993, pp. 348-357, Sept. 1993.

[2] M. Balcer, W. Hasling, and T. Ostrand, "Automatic Generation of Test Scripts from Formal Test Specifications," Proc. Third Symp. Software Testing, Analysis, and Verification, pp. 210-218, Dec. 1989.

[3] T. Ball, "On the Limit of Control Flow Analysis for Regression Test Selection," Proc. 1998 Int'l Symp. Software Testing and Analysis, Mar. 1998.

[4] S. Bates and S. Horwitz, "Incremental Program Testing Using Program Dependence Graphs," Proc. 20th ACM Symp. Principles of Programming Languages, Jan. 1993.

[5] B. Beizer, Software Testing Techniques. New York: Van Nostrand Reinhold, 1990.

[6] P. Benedusi, A. Cimitile, and U. De Carlini, "Post-Maintenance Testing Based on Path Change Analysis," Proc. Conf. Software Maintenance-1988, pp. 352-361, Oct. 1988.

[7] D. Binkley, "Semantics Guided Regression Test Cost Reduction," IEEE Trans. Software Eng., vol. 23, no. 9, Aug. 1997.

[8] Y.F. Chen, D.S. Rosenblum, and K.P. Vo, “TestTube: A System for Selective Regression Testing," Proc. 16th Int'l Conf. Software Eng., pp. 211-222, May 1994.

[9] K.F. Fischer, F. Raji, and A. Chruscicki, "A Methodology for Retesting Modified Software," Proc. National Telecomm. Conf. B-6-3, pp. 1-6, Nov. 1981.

[10] R. Gupta, M.J. Harrold, and M.L. Soffa, "An Approach to Regression Testing Using Slicing," Proc. Conf. Software Maintenance-1992, pp. 299-308, Nov. 1992.

[11] W. Harrison and C. Cook, "Insights on Improving the Maintenance Process through Software Measurement," Proc. Conf. Software Maintenance-1990, pp. 37-45, Nov. 1990.

[12] M.J. Harrold and M.L. Soffa, "An Incremental Approach to Unit Testing During Maintenance," Proc. Conf. Software Maintenance-1988, pp. 362-367, Oct. 1988.

[13] J. Hartmann and D.J. Robson, "Techniques for Selective Revalidation," IEEE Software, vol. 16, no. 1, pp. 31-38, Jan. 1990.

[14] M. Hutchins, H. Foster, T. Goradia, and T. Ostrand, "Experiments on the Effectiveness of Dataflow- and Controlflow-Based Test Adequacy Criteria," Proc. 16th Int'l Conf. Software Eng., pp. 191200, May 1994.

[15] J. Laski and W. Szermer, "Identification of Program Modifications and Its Applications in Software Maintenance," Proc. Conf. Software Maintenance-1992, pp. 282-290, Nov. 1992.

[16] J.A.N. Lee and X. He, "A Methodology for Test Selection," The J. Systems and Software, vol. 13, no. 1, pp. 177-185, Sept. 1990.

[17] H.K.N. Leung and L.J. White, "Insights Into Regression Testing," Proc. Conf. Software Maintenance-1989, pp. 60-69, Oct. 1989.

[18] H.K.N. Leung and L.J. White, "A Study of Integration Testing and Software Regression at the Integration Level," Proc. Conf. Software Maintenance-1990, pp. 290-300, Nov. 1990.

[19] H.K.N. Leung and L.J. White, "A Cost Model to Compare Regression Test Strategies," Proc. Conf. Software Maintenance-1991, pp. 201-208, Oct. 1991.

[20] T.J. Ostrand and M.J. Balcer, "The Category-Partition Method for Specifying and Generating Functional Tests," Comm. ACM, vol. 31, no. 6, June 1988.

[21] T.J. Ostrand and E.J. Weyuker, "Using Dataflow Analysis for Regression Testing," Proc. Sixth Ann. Pacific Northwest Software Quality Conf., pp. 233-247, Sept. 1988.

[22] D. Rosenblum and G. Rothermel, "An Empirical Comparison of Regression Test Selection Techniques," Proc. Int'l Workshop Empirical Studies of Software Maintenance, pp. 89-94, Oct. 1997.

[23] D.S. Rosenblum and E.J. Weyuker, "Using Coverage Information to Predict the Cost-Effectiveness of Regression Testing Strategies," IEEE Trans. Software Eng., vol. 23, no. 3, pp. 146-156, Mar. 1997.

[24] G. Rothermel and M.J. Harrold, "Empirical Studies of a Safe Regression Test Selection Technique," IEEE Trans. Software Eng., vol. 24, no. 6, pp. 401-419, June 1998.

[25] G. Rothermel and M.J. Harrold, "Analyzing Regression Test Selection Techniques," IEEE Trans. Software Eng., vol. 22, no. 8, pp. 529-551, Aug. 1996.

[26] G. Rothermel and M.J. Harrold, "A Safe, Efficient Regression Test Selection Technique," ACM Trans. Software Eng. and Methodology, vol. 6, no. 2, pp. 173-210, Apr. 1997.
[27] B. Sherlund and B. Korel, "Logical Modification Oriented Software Testing," Proc. 12th Int'l Conf. Testing Computer Software, June 1995.

[28] A.B. Taha, S.M. Thebaut, and S.S. Liu, "An Approach to Software Fault Localization and Revalidation Based on Incremental Data Flow Analysis," Proc. 13th Ann. Int'l Computer Software and Applications Conf., pp. 527-534, Sept. 1989.

[29] F. Vokolos and P. Frankl, "Pythia: A Regression Test Selection Tool Based on Textual Differencing," Proc. Third Int'l Conf. Reliability, Quality, and Safety of Software Intensive Systems (ENCRESS '97), May 1997.

[30] S.S. Yau and Z. Kishimoto, "A Method for Revalidating Modified Programs in the Maintenance Phase," Proc. 11th Ann. Int'l Computer Software and Applications Conf. (COMPSAC'87), pp. 272277, Oct. 1987.

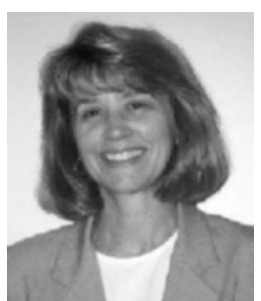

Mary Jean Harrold received the MS and MA degrees in mathematics from Marshall University and the MS and $\mathrm{PhD}$ degrees in computer science from the University of Pittsburgh. She is currently an associate professor in the College of Computing at Georgia Institute of Technology, where she leads the Aristotle Software Engineering Research Group. Her research interests include software engineering, with an emphasis on program analysis techniques that facilitate testing and maintenance tasks. Dr. Harrold is a recipient of the US National Science Foundation's National Young Investigator award. She served as program cochair of the 1997 International Conference on Software Maintenance. She is currently serving as program chair for the 2000 ACM SIGSOFT International Symposium on Software Testing and Analysis, as program cochair for the 2001 International Conference on Software Engineering, and as an associate editor for IEEE Transactions on Software Engineering. She is a member of the Computing Research Association's Committee on the Status of Women in Computing and serves as program director for the Computing Research Association's Distributed Mentor Project. She is a member of the IEEE Computer Society and the ACM.

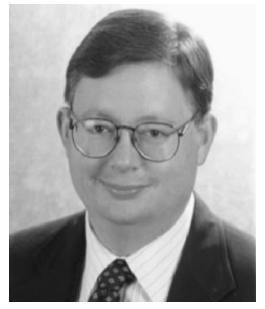

David Rosenblum received the $\mathrm{PhD}$ degree in electrical engineering in 1988 from Stanford University, where he participated in the Anna and TSL projects. He is currently an associate professor and the associate chair for computing in the Department of Information and Computer Science at the University of California, Irvine. From 1988 to 1996, he was a research member of the Technical Staff at AT\&T Bell Laboratories in Murray Hill, New Jersey. His research is currently centered on problems in the design and validation of largescale distributed component-based software systems. This work has four main thrusts: investigation of the architectural foundations of component-based software engineering, development of a theory of test adequacy for component-based systems, investigation of post-deployment monitoring techniques that exploit distributed object infrastructures, and investigation of event observation and notification technologies for wide-area networks. Dr. Rosenblum has published numerous papers on a variety of topics in software engineering. $\mathrm{He}$ recently served on the program committees for the SIGSOFT '98 Sixth International Symposium on the Foundations of Software Engineering, the 13th IEEE International Conference on Automated Software Engineering, the 1997 International Conference on Software Engineering, the 1994 and 1996 International Symposia on Software Testing and Analysis, and the Eighth International Workshop on Software Specification and Design. He is program chair for the SIGSOFT 2000 Eighth International Symposium on the Foundations of Software Engineering (FSE-8). He is the recipient of an US National Science Foundation CAREER award, a senior member of the IEEE, and a member of the ACM, the ACM SIGPLAN, the ACM SIGSOFT, the IEEE Computer Society, and IEEE Computer Society Technical Council on Software Engineering. 


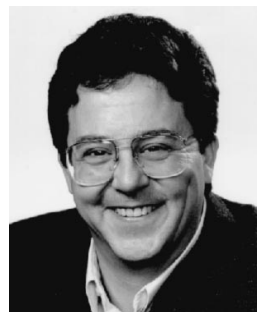

Gregg Rothermel received the BA degree in philosophy from Reed College, the MS degree in computer science from the State University of New York in Albany, and the PhD degree in computer science from Clemson University. He is currently an assistant professor in the Computer Science Department of Oregon State University. His research interests include software engineering and program analysis with an emphasis on the application of program analysis techniques to problems in software maintenance and testing. Dr. Rothermel is a recipient of the US National Science Foundation's Faculty Early Career Development Award and of the Oregon State University College of Engineering's Engelbrecht Young Faculty Award. $\mathrm{He}$ is serving on the program committees for the 2000 ACM SIGSOFT International Symposium on Software Testing and Analysis, the 2000 International Conference on Software Engineering, the 2001 International Conference on Software Engineering, the SIGSOFT 2000 Eighth International Symposium on the Foundations of Software Engineering (FSE-8), and the 2000 International Conference in Software Maintenance. He is a member of the IEEE Computer Society, the ACM, the ACM SIGSOFT, and the ACM SIGPLAN.

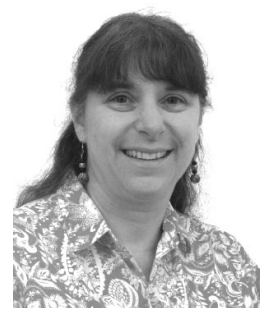

Elaine Weyuker received the BA degree in mathematics from the State University of New York in Binghamton, the MSE degree from the Moore School of Electrical Engineering, University of Pennsylvania, and the PhD degree in Computer Science from Rutgers University. She is currently a technology leader at AT\&T Labs-Research in Florham Park, New Jersey. Before moving to AT\&T Labs in 1993, she was a professor of computer science at the Courant Institute of Mathematical Sciences of New York University, New York, where she had been on the faculty since 1977. Prior to New York University, she was on the faculty of the City University of New York. Her research interests are in software engineering, particularly, software testing and reliability, and software metrics. She is also interested in the theory of computation and is the author of a book (with Martin Davis and Ron Sigal), Computability, Complexity, and Languages, second ed., published by Academic Press. Dr. Weyuker is a Fellow of the ACM and a senior member of the IEEE. She is a member of the editorial boards of ACM Transactions on Software Engineering and Methodology (TOSEM), the Empirical Software Engineering Journal, and an advisory editor of the Journal of Systems and Software. She has been the Secretary/Treasurer of ACM SIGSOFT, on the executive board of the IEEE Computer Society Technical Committee on Software Engineering, and was an ACM National Lecturer. 